\title{
Predictive Power of Body Visceral Adiposity Index, Body Adiposity Index and Body Mass Index for Type 2 Diabetes in Qatari Population Aysha Al-Khalaqi ${ }^{1}$, Fatima Al-Naimi ${ }^{1}$, Rouda Qassmi ${ }^{1}$ Supervised by: Prof. Hiba Bawadi ${ }^{1}$ ${ }^{1}$ Human Nutrition Department, College of Health Sciences, Qatar University, Doha, Qatar
}

\section{INTRODUCTION}

Diabetes mellitus (DM) is the ninth leading cause of death worldwide and approximately $90 \%$ of cases are $\mathrm{T}^{2} \mathrm{D}^{1}$. In Qatar, it is estimated that by 2050, one Qatari in every four will have diabetes ${ }^{2}$. Evidence shows that higher body fat is associated with increased risk for severa metabolic disorders ${ }^{3}$. Since the 1990 s, BMI has been used to define underweight, overweight, and obesity. Many studies have questioned the utility of BMI in weig lassification as it cannot differentiate between lean mass and fat mass ${ }^{4}$. BAl was first introduced in 2011, based on anthropometric measurements to evaluate adult body fat (BF) and body fat percentage (BF\%). BAl has been noted for its low cost and ease of administration ${ }^{5}$. However, VA seems to be a better predictor for metabolic disorders associated with insulin resistance than a single anthropometric index. Although both VAI and BAI can predict the risk of metabolic diseases such as T2D, it is not known which of these is a better predictor of T2D in Qatari adults. Therefore, the aim of this study was to investigate the effectiveness of VAI and BAI in predicting the risk of T2D in the Qatari adult population.

\section{METHODOLOGY}

\section{Study Design and Population}

This study used data from the Qatar Biobank. A random sample of 1103 adults over 20 years old, who had lived in Qatar for more than 15 years, was included. Sociodemographic data, lifestyle factors and dietary habits were collected by a self-administrated questionnaire. Data regarding health condition, family history of disease and medication use was collected by a registered nurse through face-to-face interviews. All the study protocols were approved by the Qatar Biobank Institutional Review Board.

\section{Independent Variable}

BMI was calculated as weight in $\mathrm{kg}$ divided by standing height in $\mathrm{m}^{2}$

BAI was calculated as: BAI $=$ hip circumference $(\mathrm{cm}) /$ height $(\mathrm{m})^{1.5}-18$.

VAl scores for men and women were calculated with the following formulas:

Males: VAI

$$
=\frac{W C(\mathrm{~cm})}{39.68+(1.88 \times B M I)} \times\left(\frac{T G(\mathrm{mmol} / \mathrm{L})}{1.03}\right) \times\left(\frac{1.31}{H D L-C(\mathrm{mmol} / \mathrm{L})}\right)
$$$$
=\frac{W C(\mathrm{~cm})}{36.58+(1.89 \times B M I)} \times\left(\frac{T G(\mathrm{mmol} / \mathrm{L}}{0.81}\right) \times\left(\frac{1.52}{H D L-C(\mathrm{mmol} / \mathrm{L})}\right)
$$

\section{Anthropometric Measurements}

Body weight, height and waist circumference (WC) were measured according to the standard methods. Body fat and visceral fat were measured by iDXA scan.

\section{Biochemical measurements}

Blood samples were collected after at least 8 hours of overnight fasting. Measurements included fasting blood glucose (FPG) and HbA1c as well as Blood lipid profiles of total cholesterol (TC), total triglycerides (TG), low-density lipoprotein-cholesterol (LDL-C) and HDL-C were obtained.

\section{Statistical Analysis}

Chi square test was used to compare the differences between genders for categorical variables and t-test for continuous variables. Multivariable logistic regression was used to assess the association between different measures of obesity ( $\mathrm{z}$ scores of VAl, BAI, and BMI) and diabetes. All the analyses were performed by using STATA 16 . Statistical significance was considered when $p<0.05$ (two sided).

\section{ABSTRACT}

Background: The prevalence of type 2 diabetes (T2D) has increased recently in Qatar. Body mass index (BMI) is a predictor of T2D in many populations. However, BMI is based on height and weight measurements and not on body adiposity. Therefore, the utility of BMI for predicting the risk of T2D has been questioned, and visceral adiposity (VAI) appears to be a better predictor of T2D.

Objective: This study aimed to assess the relative effectiveness of visceral adiposity index (VAI) and body adiposity index (BAI), in comparison with body mass index (BMI), for T2D among Qatari adults.

Methodology: A random sample of 1103 adult Qatari nationals over 20 years old were included in this study; this data was obtained from the Qatar National Biobank. We performed a multivariate logistic regression to examine the association between VAI, BAI, BMI, and T2D, and computed zscores for VAI, BAI and BMI.

Result: VAl z-scores showed the strongest association with the risk of T2D (OR, 1.44; 95\% Cl: 1.24-1.68) compared with the z-scores for BAI (OR, 1.15; 95\% Cl: 0.93-1.43) and BMI (OR,

1.33; $95 \% \mathrm{Cl}: 1.11-1.59)$. Subgroup analyses indicated that the association was stronger between VAl and T2D in Qatari women than in men.

Conclusion: VAI was a strong and independent predictor of T2D among the Qatari adult population. Therefore, VAl could be a useful tool for predicting the risk of T2D among Qatari adults.

Keywords: Body adiposity index, Body mass index,

Qatar Biobank, Type 2 diabetes, Visceral adiposity

index

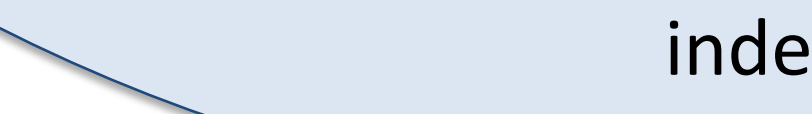

\section{RESULTS}

Table 1 shows the characteristics of the study sample by diabetes status. More than $50 \%$ of participants with diabetes were women, while non-diabetic participants were mostly men. Moreover, after adjusting for age and gender, the VAl z-score was directly associated with the prevalence of diabetes while BMI z-scores showed lower association. With a further adjustment for education and physical activity, the VAl zscore was more strongly associated with the prevalence of diabetes compared to the $\mathrm{BMI}$ z-score. There was no relationship between BAl z-score and the prevalence of diabetes (Table 2)

Table 1. Sample characteristics by diabetes.

\begin{tabular}{|l|l|l|l|l|}
\hline & Total & $\mathrm{No}$ & Yes & P-value \\
\hline & $\mathrm{N}=1103$ & $\mathrm{~N}=916$ & $\mathrm{~N}=187$ & \\
\hline Sex & & & & 0.14 \\
Male & $567(51.4 \%)$ & $480(52.4 \%)$ & $87(46.5 \%)$ & \\
Female & $536(48.6 \%)$ & $436(47.6 \%)$ & $100(53.5 \%)$ & \\
Age (years) & $39.7(11.2)$ & $37.6(10.4)$ & $50.2(9.1)$ & $<0.001$ \\
Education & & & $<0.001$ \\
Low & $125(11.3 \%)$ & $68(7.4 \%)$ & $57(30.5 \%)$ & \\
Medium & $338(30.7 \%)$ & $287(31.4 \%)$ & $51(27.3 \%)$ & \\
High & $639(58.0 \%)$ & $560(61.2 \%)$ & $79(42.2 \%)$ & \\
BMI (kg/m $\left.{ }^{2}\right)$ & $29.3(5.8)$ & $28.8(5.6)$ & $31.8(5.8)$ & $<0.001$ \\
BMl categories & & & & $<0.001$ \\
Normal & $243(22.0 \%)$ & $226(24.7 \%)$ & $17(9.1 \%)$ & \\
Overweight & $399(36.2 \%)$ & $340(37.1 \%)$ & $59(31.6 \%)$ & \\
Obese & $461(41.8 \%)$ & $350(38.2 \%)$ & $111(59.4 \%)$ & \\
Waist circumference $(\mathrm{cm})$ & $89.7(13.8)$ & $88.0(13.5)$ & $97.9(12.7)$ & $<0.001$ \\
Body adiposity index & $32.7(6.9)$ & $32.3(6.6)$ & $34.6(7.6)$ & $<0.001$ \\
Visceral adiposity index & $1.7(1.6)$ & $1.6(1.5)$ & $2.5(1.8)$ & $<0.001$ \\
\hline
\end{tabular}
Data is presented as mean (SD) for continuous measures, and $n(\%)$ for categorical measures.

Table 2. Association of VAI and BAI with diabetes among Qatari adults.

\begin{tabular}{l|c|c|c|c|c|c|}
\hline \multirow{2}{*}{} & \multicolumn{2}{|c|}{ VAl z-score } & \multicolumn{2}{c|}{ BAI z-score } & \multicolumn{2}{c|}{ BMI z-score } \\
\cline { 2 - 7 } & $\mathrm{OR}(95 \% \mathrm{CI})$ & $\mathrm{P}$ & $\mathrm{OR}(95 \% \mathrm{Cl})$ & $\mathrm{P}$ & $\mathrm{OR}(95 \% \mathrm{Cl})$ & $\mathrm{P}$ \\
\hline Model 1 & $1.44(1.24-1.68)$ & $<0.001$ & $1.15(0.93-1.43)$ & 0.203 & $1.33(1.11-1.59)$ & 0.002 \\
Model 2 & $1.39(1.19-1.63)$ & $<0.001$ & $1.09(0.87-1.36)$ & 0.445 & $1.28(1.07-1.54)$ & 0.008 \\
Model 3 & $1.38(1.18-1.61)$ & $<0.001$ & $1.08(0.86-1.35)$ & 0.502 & $1.27(1.06-1.53)$ & 0.011 \\
\hline
\end{tabular}

Subgroup analyses suggested that there was a significant interaction between VA and diabetes with gender and age (Figure 1). The association between BAI z-score and diabetes was not significant when stratified by gender, age, education and BM (Figure 2). Furthermore, subgroup analyses suggested that there was a significant interaction between BMI and diabetes with gender, age and education (Figure 3).

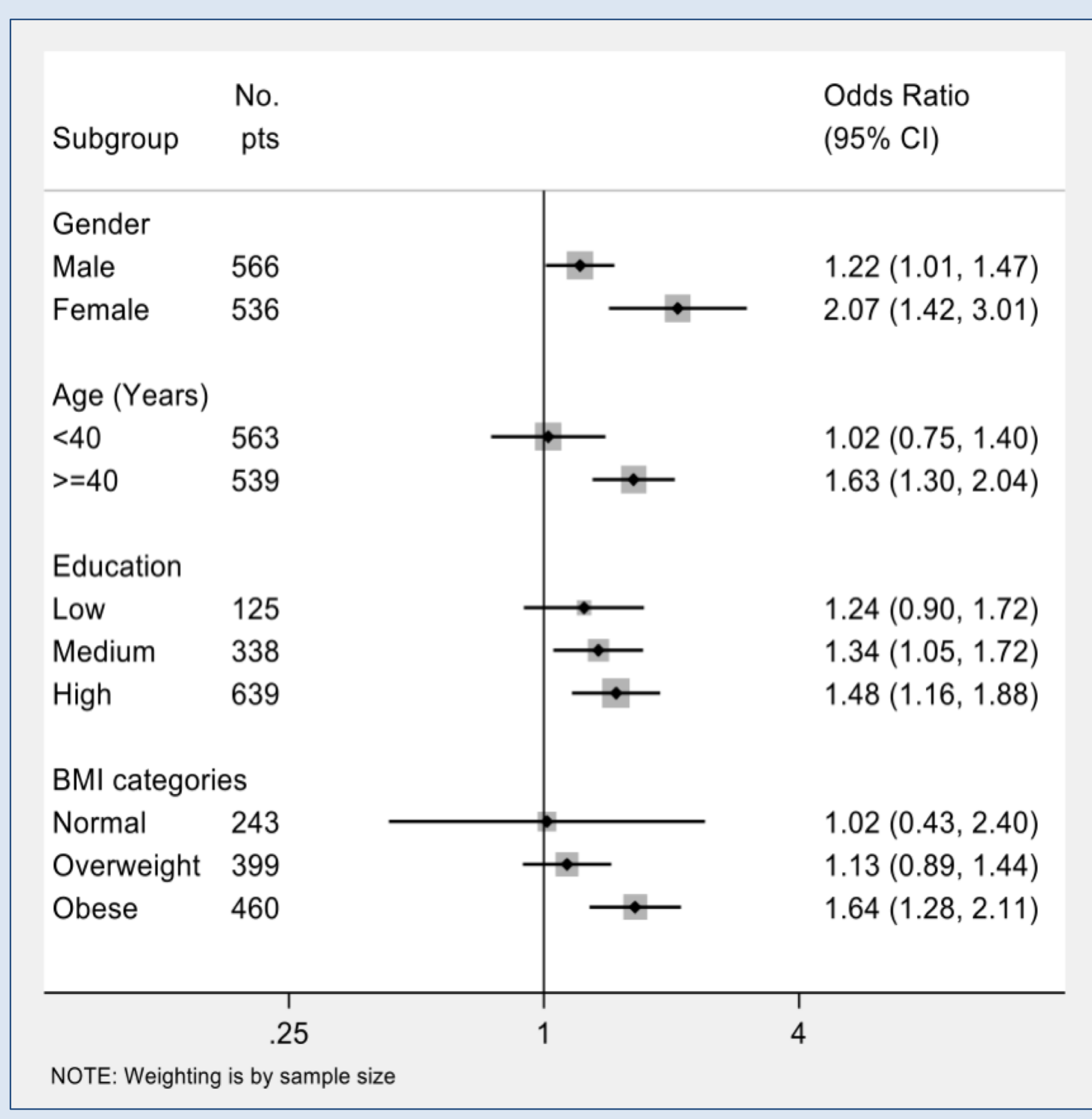

Figure 1. Subgroup analysis of the association between VAI z-score and diabetes. Values adjusted for age, gender, education and physical activity.

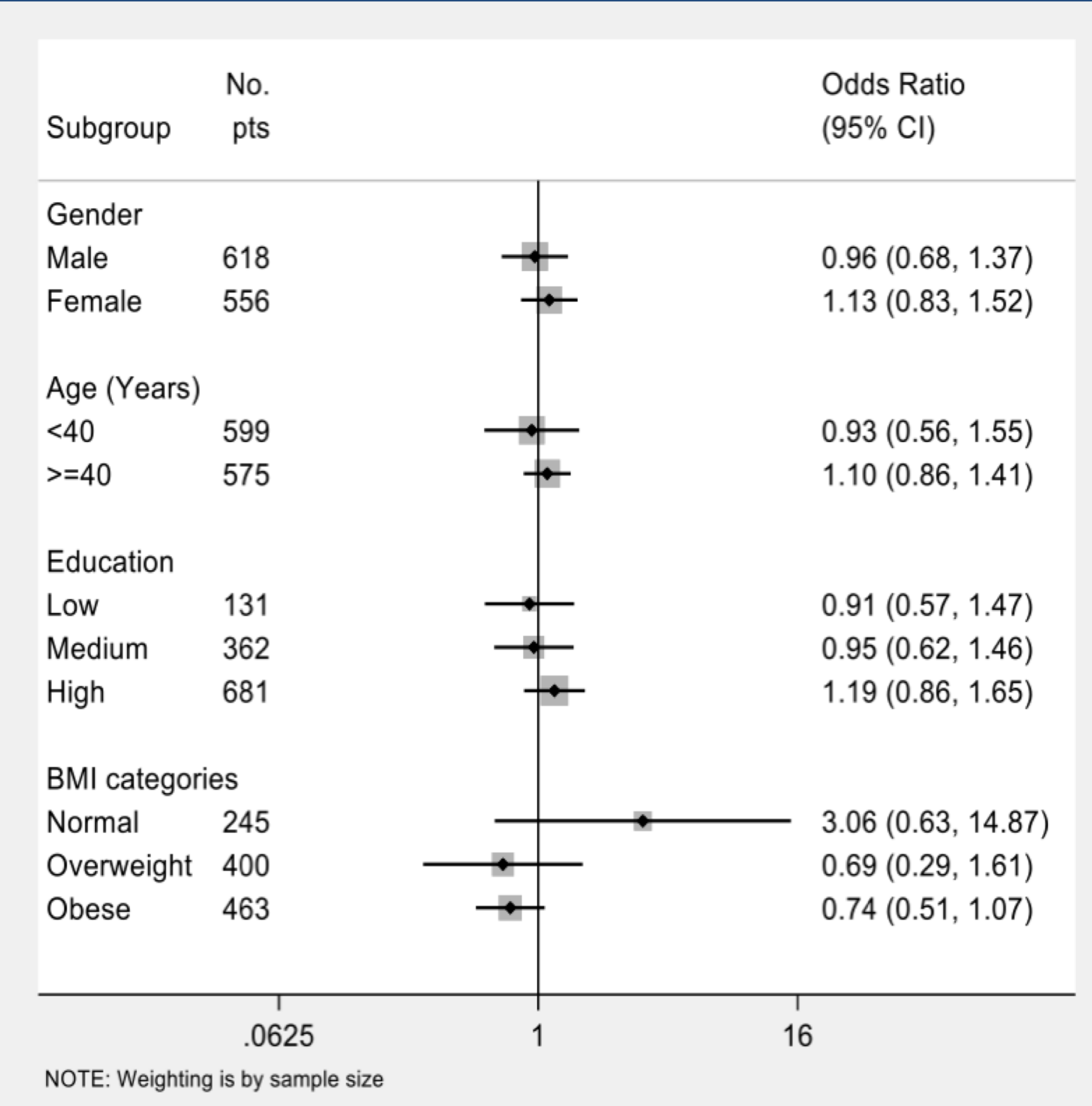

Figure 2. Subgroup analysis of the association between BAI z-score and diabetes. Values adjusted for age, gender, education and physical activity.

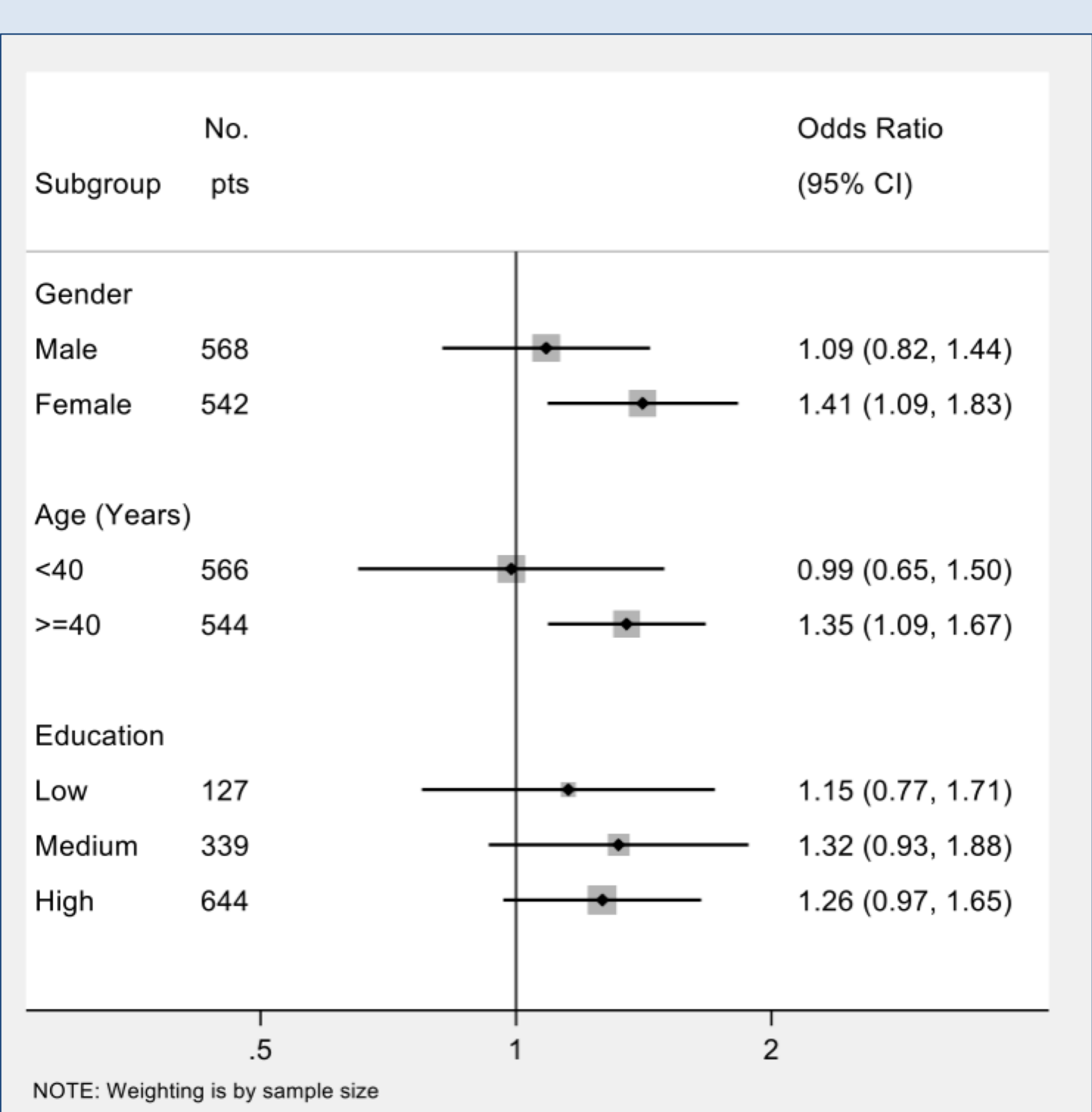

igure 3. Subgroup analysis of the association between BMI z-score and diabetes. Values adjusted for age, gender, education and physical activity.

\section{CONCLUSION}

In conclusion, our study indicated that VAl is a strong and independent predictor of T2D among the Qatari population. The predictive ability of VAl is superior to that of BMI and BAI. Therefore, VAl could be a useful tool for the prediction of the risk of T2D among Qataris. Nevertheless, if VAI were not available, BMI is still known to be non-invasive and the most applicable compared with other measurements.

\section{ACKNOWLEDGEMENTS}

Acknowledgement to Qatar Biobank for providing the data. The publication of this article was funded by the Qatar National Library. This research is funded by Qatar University.

1. Zheng Y, Ley SH, Hu FB. Global aetiology and epidemiology of type 2 diabetes mellitus and its complications. Nature Reviews Endocrinology 2018; 14(2): 88.
2. Awad SF, O'Flaherty M, Critchley J, Abu-Raddad L. Forecasting the burden of type 2 diabetes mellitus in Qatar to 2050: a novel modeling approach. Diabetes Research and Clinical Practice 2018; 137: 100-8.

REFERENCES 3. Ding C, Chan Z, Chooi YC, et al. Visceral adipose tissue tracks more closely with metabolic dysfunction than intrahepatic triglyceride in lean Asians without diabetes. Journal of Applied Physiology 2018; 125(3): 909-15. 4. Javed A, Jumean M, Murad MH, et al. Diagnostic performance of body mass index to identify obesity as defined by body adiposity in children and adolescents: a systematic review and meta-analysis. Pediatric Obesity 2015: 10(3): 234-44. 5. Bergman RN, Stefanovski D, Buchanan TA, et al. A better index of body adiposity. Obesity (Silver Spring) 2011; 19(5): 1083-9. 